\title{
FATORES ASSOCIADOS AO RELATO DE EPISIOTOMIA EM PARTURIENTES ATENDIDAS EM UM HOSPITAL DO OESTE CATARINENSE
}

\section{FACTORS ASSOCIATED WITH THE REPORT OF EPISIOTOMY IN PARTURIENTS A HOSPITAL IN THE WEST SANTA CATARINA}

\section{FACTORES ASOCIADOS AL INFORME DE EPISIOTOMÍA EN PARTURIENTE DE UN HOSPITAL EN EL OESTE SANTA CATARINA}

Joice Moreira Schmalfuss ${ }^{1}$, Maira Rossetto ${ }^{2}$, Lilian Baseggio3 ${ }^{3}$, Vitoria Radichewski ${ }^{4}$, Joanna d'Arc Lyra Batista ${ }^{5}$.

\section{RESUMO}

Objetivo: identificar os fatores associados ao relato de episiotomia em parturientes atendidas em um hospital referência do oeste catarinense. Métodos: trata-se de estudo transversal realizado com mulheres submetidas a parto vaginal hospitalar. A coleta de dados ocorreu diariamente, entre agosto e setembro de 2016, por meio de questionário padronizado e informações complementadas pelo prontuário e cartão pré-natal. A variável dependente foi o relato da realização de episiotomia. Os dados foram submetidos à análise bivariada e multivariada. Resultados: foram incluídas no estudo 136 participantes. A episiotomia foi relatada por 84 (61,8\%) das mulheres. Ser nulípara e ter tido número excessivo de toques vaginais ficaram associados à realização de episiotomia no modelo final de regressão logística multivariada. Conclusão: apesar de esforços no sentido de diminuir as excessivas intervenções no processo de parturição, a realidade da assistência obstétrica brasileira merece atenção e profundas mudanças. É necessário que intervenções não recomendadas e danosas como a episiotomia estejam em consonância com as boas práticas obstétricas e que intervenções realizadas de forma excessiva, como os toques vaginais, sejam evitadas. Descritores: Saúde da Mulher; Parto Normal; Períneo; Episiotomia; Paridade.

\begin{abstract}
Objetive: to identify factors associated with the episiotomy report in parturients attended at a reference hospital in the West of Santa Catarina. Methods: it is a cross-sectional study with women undergoing vaginal delivery in the hospital. Data collection was performed daily, between August and September 2016, through a standardized questionnaire and information complemented with consultation to the medical records and prenatal card. The dependent variable was the report of the episiotomy. Data were submitted to bivariate and multivariate analysis. Results: one hundred thirty six participants were included in the study. Episiotomy was reported by 84 (61.8\%) women. Being primiparous and reporting an excessive number of vaginal touches were associated with episiotomy in the final multivariate logistic regression model. Conclusion: despite efforts to reduce excessive interventions in the parturition process, the reality of Brazilian obstetric care deserves attention and profound changes. It is necessary that nonrecommended and harmful interventions such as the episiotomy are in accordance with good obstetrical practices and that excessive interventions, such as vaginal touches, must be avoided.

Descriptors: Women's Health; Natural Childbirth; Perineum; Episiotomy; Parity.
\end{abstract}

\section{RESUMEN}

Objetivo: identificar los factores asociados con el informe de episiotomía en parturientas tratadas en un hospital de referencia en el oeste de Santa Catarina. Métodos: es um estudio transversal realizado con mujeres sometidas a parto vaginal en el hospital. La recolección de datos se produjo diariamente, entre agosto y septiembre de 2016, por medio de un cuestionario padronizado e información complementada por el prontuario y la tarjeta prenatal. La variable dependiente fue el informe de episiotomía. Los datos se sometieron a análisis bivariados y multivariados. Resultados: 136 participantes fueron incluídas en el estudio. La episiotomía fue reportada por $84(61,8 \%)$ de las mujeres. Ser nulípara y tener um número excesivo de toques vaginales se asociaron con episiotomía en el modelo final de regresión logística multivariada. Conclusión: a pesar de los esfuerzos para reducir las intervenciones excesivas en el proceso de parto, la realidad de la atención obstétrica brasileña merece atención y câmbios profundos. Las intervenciones no recomendadas y nocivas, como la episiotomía, deben estar em línea con las buenas prácticas obstétricas y deben evitarse las intervenciones excesivas, como los toques vaginales.

Descriptores: Saúde da Mulher; Parto Normal; Períneo; Episiotomia; Paridad.

${ }^{1}$ Enfermeira obstetra, mestre e doutoranda em Ciências da Saúde. Docente da Universidade Federal da Fronteira Sul (UFFS) - Campus Chapecó/SC. ${ }^{2}$ Enfermeira, mestre e doutora em Enfermagem. Docente da Universidade Federal da Fronteira Sul (UFFS) - Campus Chapecó/SC. ${ }^{3}$ Estudante do curso de Medicina da Universidade Federal da Fronteira Sul (UFFS) - Campus Chapecó/SC. ${ }^{4}$ Estudante do curso de Medicina da Universidade Federal de Santa Catarina (UFSC) - Florianópolis/SC. ${ }^{5}$ Biomédica, mestre, doutora em Saúde Pública e pós-doutora em Medicina Tropical e em Epidemiologia. Docente da Universidade Federal da Fronteira Sul (UFFS) - Campus Chapecó/SC.

Como citar este artigo:

Schmalfuss JM, Rossetto M, Baseggio L, et al. Fatores associados ao relato de episiotomia em parturientes atendidas em um hospital do oeste catarinense. Revista de Enfermagem do Centro Oeste Mineiro. 2019;9:e3294. [Access ]; Available in: DOI: http://dx.doi.org/10.19175/recom.v9i0.3294 


\section{INTRODUÇÃO}

A assistência obstétrica no Brasil tem sido objeto de estudo, nos últimos anos, a fim de aprimorar a atenção direcionada às gestantes, parturientes, puérperas e seus recém-nascidos, nos serviços de saúde. Tal assistência ainda é conhecida por suas metas de celeridade e oportunismo mediante a tentativa de controle temporal do trabalho de parto e parto, desrespeito à autonomia da mulher no processo de parturição e agilidade do nascimento do bebêe ${ }^{(1-2)}$. Muitas das intervenções realizadas desconsideram as evidências científicas e são baseadas, majoritariamente, pelas rotinas adotadas pelos serviços de saúde e pelas decisões médicas, gerando um potencial iatrogênico à mulher e seu filho ${ }^{(1-2)}$.

Algumas intervenções utilizadas de forma rotineira, principalmente em nulíparas, tal como o rompimento artificial das membranas amnióticas, a administração de ocitocina, no primeiro período clínico do trabalho de parto (dilatação); a pressão do fundo uterino para o nascimento do bebê (manobra de Kristeller) e o alargamento do períneo provocado por uma incisão cirúrgica com tesoura ou lâmina de bisturi (episiotomia) no período expulsivo ${ }^{(2-3)}$, podem ser danosas para o binômio, além de se configurarem em violência obstétrica.

Em relação à episiotomia, ressalta-se que sua prática rotineira não só carece de base científica como pode trazer complicações intra e pós-operatórias ${ }^{(4)}$, o que indica que seu uso deve ser bastante seletivo. Os estudos que avaliam os fatores de risco para a ocorrência da episiotomia são frequentes na literatura internacional, sendo escassos com mulheres brasileiras. Eles mostram algumas variáveis que se configuram em risco para a realização da episiotomia, sendo que as mais observadas são: a nuliparidade, a litotomia, o maior peso ao nascer do recém-nascido, a maior idade materna e a instrumentalização do parto ${ }^{(5-7)}$.

$\mathrm{Na}$ atualidade, as taxas de episiotomia seguem altas em todo o mundo. Na França ${ }^{(8)}$ é equivalente a $19,9 \%$, na Colômbia ${ }^{(9)}$ a $30,48 \%$ e na Espanha $^{(7)}$ a $50 \%$. No Brasil, as taxas de episiotomia são de $53,5 \%{ }^{(10)}$, variando de acordo com a região do país. Um estudo que objetivou descrever práticas e intervenções utilizadas durante o trabalho de parto e parto e fatores associados a essas práticas em puérperas no estado de Sergipe, evidenciou a ocorrência de episiotomia em $43,9 \%$ das mulheres incluídas no estudo $^{(11)}$.
Tendo em vista que as taxas de episiotomia são bastante altas no Brasil e que sua realização necessita de caráter seletivo, torna-se imprescindível identificar os fatores de risco associados a essa intervenção obstétrica de forma a contribuir para a avaliação crítica acerca da necessidade de seu uso, qualificando a assistência profissional no parto. Ademais, esse estudo pode contribuir para a produção de conhecimento brasileiro sobre a prática da episiotomia.

Diante da questão premente de produzir dados acerca da realidade do sul do Brasil sobre a realização de intervenções desnecessárias no parto, neste trabalho, objetivou-se identificar os fatores associados ao relato de episiotomia em parturientes atendidas em um hospital referência do oeste catarinense.

\section{MÉTODOS}

Foi realizado um estudo transversal, com o objetivo de caracterizar todos os partos atendidos em um hospital de referência, no município de Chapecó, Santa Catarina, no sul do Brasil. Foram critérios de inclusão: ser puérpera, estar nas primeiras 48 horas de pós-parto e estar internada na maternidade. Os critérios de exclusão foram: ter idade inferior a 18 anos, não falar português e estar em condições clínicas que impossibilitassem a participação no estudo. Ressalta-se que se estabeleceu um critério de exclusão relacionado ao idioma em virtude do oeste Catarinense ter recebido, nos últimos anos, muitos imigrantes haitianos e estes apresentarem dificuldades de compreensão e de fala da língua portuguesa.

A coleta de dados foi realizada, diariamente, entre agosto e setembro de 2016, por meio de questionário padronizado aplicado pelas pesquisadoras na beira do leito. 0 questionário foi elaborado especialmente para essa pesquisa e abordou informações referentes aos antecedentes obstétricos, ao parto atual, ao(s) recém-nascido(s) e ao pré-natal. Informações foram complementadas com consulta ao prontuário e cartão pré-natal de cada puérpera entrevistada. Para evitar o viés de seleção, as participantes da pesquisa foram selecionadas por meio de plantão dos entrevistadores, durante todos os dias da semana, sendo todo o período estudado coberto.

No presente artigo, analisaram-se apenas os partos vaginais dentre a amostra total do estudo base. Neste trabalho, a variável 
dependente foi o relato da realização de episiotomia referida pela entrevistada. A pergunta sobre a realização da episiotomia foi feita em linguagem simples indagando a mulher sobre a realização de um corte na região da vagina. As variáveis de exposição foram selecionadas e adaptadas com base em estudos prévios $^{(5,7)}$ e no questionário do estudo Nascer no Brasil $^{(12)}$.

A variável "toques repetitivos" foi definida de acordo com a percepção da puérpera ao responder a seguinte pergunta: Foram realizados exames de toque repetitivos durante o trabalho de parto?

Dados coletados no Departamento de Informática do Sistema Único de Saúde (DATASUS) mostraram que Chapecó contabilizou 3.183 nascidos vivos em 2013 (ano em que o projeto foi construído), sendo que 74 nascimentos foram decorrentes de gestação dupla, nenhum de gestação tripla e três nascimentos por tipo de gestação ignorada. Considerando estes dados, levantou-se o número de 3.146 puérperas para o ano de 2013. A partir desses achados e de percentuais de episiotomia de acordo com a exposição "posição de litotomia durante 0 parto" descritos na literatura ${ }^{(7)}$, resultou-se em um tamanho de amostra total de 142 puérperas, considerando o poder do estudo de $80 \%$ e um nível de confiança de $95 \%$.

Regressão logística multivariada backward foi utilizada para identificar os fatores associados à episiotomia autorreferida. As variáveis associadas ao desfecho com $p \leq 0,20$ na análise bivariada foram incluídas no modelo multivariado. A análise se deu por meio do programa STATA versão 12.
A pesquisa base foi aprovada pelo Comitê de Ética em Pesquisa da Universidade Federal da Fronteira Sul, sob parecer de número $1.575 .071 \mathrm{e}$ todas as entrevistadas assinaram o Termo de Consentimento Livre e Esclarecido antes de concederem a entrevista.

\section{RESULTADOS}

Das puérperas que se adequaram aos critérios de inclusão, três recusaram participar do estudo. No total, foram entrevistadas 343 mulheres no período entre 10 de agosto e 30 de setembro do ano de 2016, para o estudo base. Destas, 137 (39,9\%) foram submetidas a parto vaginal. Em seis partos houve relato sobre a necessidade de intervenções para a extração do feto com o uso de fórceps. Uma das participantes foi excluída da análise, por não saber responder, quando questionada, durante a entrevista, se foi submetida à episiotomia. Dessa forma, a amostra final deste estudo foi composta por 136 puérperas. A episiotomia foi relatada por 84 $(61,8 \%)$ mulheres, enquanto que $67(49,3 \%)$ mencionaram ter sofrido a manobra de Kristeller, durante o período expulsivo.

A idade média das mulheres foi de 26,7 anos (18-42), 14\% residiam em municípios vizinhos à Chapecó, a maioria $(60,3 \%)$ apresentou cor da pele branca e todas realizaram acompanhamento pré-natal. A maioria das entrevistadas não apresentou eventos de risco, durante o período gestacional; teve um acompanhante de sua escolha durante o trabalho de parto, parto e/ou pós-parto; teve partos que ocorreram a termo e que resultaram em nascimentos de bebês do sexo masculino, conforme se verifica na Tabela 1.

Tabela 1. Características sociodemográficas, variáveis clínicas e laboratoriais, variáveis relacionadas ao serviço de saúde e às puérperas e sexo do bebê em parturientes atendidas em um hospital referência do Oeste Catarinense, 2016.

\begin{tabular}{ll}
\hline \multicolumn{1}{c}{ Características } & Total de puérperas $\mathbf{n}(\%)$ \\
\hline Sociodemográficas & \\
Idade & $26,7(18-42)$ \\
Cor da pele branca & $82(60,3)$ \\
Reside em Chapecó & $117(86,0)$ \\
Variáveis clínicas e laboratoriais & \\
Eventos de risco durante gestação* & $11(8,1)$ \\
Idade gestacional média & \\
Pré-termo (menor que 37 semanas de gestação) & $10(7,4)$ \\
Termo (entre 37 e 41 semanas e 6 dias) & $126(92,6)$ \\
HIV reagente & $01(0,7)$ \\
VDRL reagente & $02(1,5)$
\end{tabular}




\begin{tabular}{ll} 
Variáveis relacionadas ao serviço de saúde & \\
Realização de pré-natal & $136(100 \%)$ \\
Variáveis relacionadas às puérperas & \\
Permitir acompanhante & $134(98,5)$ \\
$\quad$ Acompanhante no pré-parto & $132(97,1)$ \\
Acompanhante no expulsivo & $133(97,8)$ \\
$\quad$ Acompanhante após parto & $133(97,8)$ \\
Durante a gestação se sentiu & \\
$\quad$ Inferiorizada/Diminuída & $03(2,2)$ \\
Intimidada & $03(2,2)$ \\
$\quad$ Desprezada & $03(2,2)$ \\
$\quad$ Com medo & $39(28,7)$ \\
Sexo do bebê & \\
$\quad$ Masculino & $76(55,9)$ \\
\hline \hline
\end{tabular}

Fonte: Elaborada pelas autoras.

*Diabetes gestacional, pressão alta, placenta prévia, eclâmpsia e/ou cerclagem.

No modelo bivariado, as seguintes variáveis obtiveram $\mathrm{p} \leq 0,20$ e foram selecionadas para $\mathrm{o}$ modelo multivariado: idade inferior a 30 anos, não ter gravidez anterior (nulípara), estar em posição litotômica, durante o período expulsivo, ter sofrido manobra de Kristeller e ter relatado excesso de toques vaginais, durante o processo de parturição, de acordo com o observado na Tabela 2.

Tabela 2. Análise bivariada dos fatores associados ao relato de realização de episiotomia em parturientes atendidas em um hospital referência do Oeste Catarinense, 2016.

\begin{tabular}{|c|c|c|c|c|}
\hline & \multicolumn{2}{|c|}{ Episiotomia autorreferida } & \multirow[t]{2}{*}{ OR (IC 95\%) } & \multirow[t]{2}{*}{$p$} \\
\hline & $\begin{array}{l}\text { Não } \\
\text { n (\%) }\end{array}$ & $\begin{array}{l}\text { Sim } \\
\text { n (\%) }\end{array}$ & & \\
\hline \multicolumn{5}{|l|}{ Variáveis Biológicas } \\
\hline \multicolumn{5}{|l|}{ Grupo Etário da puérpera } \\
\hline 18 a 29 anos & $31(34,4)$ & $59(65,6)$ & 1,0 & \\
\hline 30 ou mais anos & $21(45,6)$ & $25(54,4)$ & $0,63(0,30-1,29)$ & 0,205 \\
\hline \multicolumn{5}{|l|}{ Variáveis Socioeconômicas } \\
\hline \multicolumn{5}{|l|}{ Cidade de residência } \\
\hline Chapecó & $44(37,6)$ & $73(62,4)$ & 1,0 & \\
\hline Outra cidade & $08(42,1)$ & $11(57,9)$ & $0,83(0,31-2,22)$ & 0,708 \\
\hline \multicolumn{5}{|l|}{ Cor da pele autorreferida } \\
\hline Branca & $34(41,5)$ & $48(58,5)$ & 1,0 & \\
\hline Preta, parda, amarela, ou indígena & $17(32,1)$ & $36(67,9)$ & $1,50(0,73-3,10)$ & 0,273 \\
\hline \multicolumn{5}{|l|}{ Gravidez anterior } \\
\hline Sim (multípara) & $44(51,8)$ & $41(48,2)$ & 1,0 & \\
\hline Não (nulípara) & $8(15,7)$ & $43(84,3)$ & $5,77(2,42-13,7)$ & 0,000 \\
\hline \multicolumn{5}{|l|}{ Variáveis relacionadas ao parto } \\
\hline \multicolumn{5}{|l|}{ Uso de fórceps } \\
\hline Não & $51(39,2)$ & $79(60,8)$ & 1,0 & \\
\hline Sim & $01(16,7)$ & $05(83,3)$ & $3,23(0,37-28,4)$ & 0,291 \\
\hline \multicolumn{5}{|l|}{ Financiamento do parto } \\
\hline Privado & $01(16,7)$ & $05(83,3)$ & 1,0 & \\
\hline Público & $51(39,5)$ & $78(60,5)$ & $0,31(0,35-2,69)$ & 0,286 \\
\hline \multicolumn{5}{|c|}{ Acompanhante durante período expulsivo } \\
\hline Não & $01(33,3)$ & $02(66,7)$ & 1,0 & \\
\hline Sim & $38(38,3)$ & $82(61,6)$ & $0,80(0,07-9,09)$ & 0,860 \\
\hline \multicolumn{5}{|c|}{$\begin{array}{l}\text { Livre movimentação durante o trabalho de } \\
\text { parto }\end{array}$} \\
\hline Sim & $47(40,2)$ & $70(59,8)$ & 1,0 & \\
\hline Não & $05(26,3)$ & $14(73,7)$ & $1,88(0,63-5,57)$ & 0,255 \\
\hline \multicolumn{5}{|c|}{ Posição de litotomia durante expulsivo } \\
\hline Não & $11(57,9)$ & $08(42,1)$ & 1,0 & \\
\hline Sim & $41(35,0)$ & $76(65,0)$ & $2,55(0,95-6,84)$ & 0,063 \\
\hline \multicolumn{5}{|l|}{ Manobra de Kristeller } \\
\hline Não & $30(43,5)$ & $39(56,5)$ & 1,0 & \\
\hline Sim & $22(32,8)$ & $45(67,2)$ & $1,57(0,78-3,16)$ & 0,203 \\
\hline
\end{tabular}




\begin{tabular}{|c|c|c|c|c|}
\hline \multicolumn{5}{|c|}{$\begin{array}{l}\text { Contato pele a pele com bebê logo que } \\
\text { nasceu }\end{array}$} \\
\hline Sim & $48(38,7)$ & $76(61,3)$ & 1,0 & \\
\hline Não & $04(33,3)$ & $08(66,7)$ & $1,26(0,36-4,42)$ & 0,715 \\
\hline \multicolumn{5}{|c|}{$\begin{array}{l}\text { Mínimo de seis consultas durante o pré- } \\
\text { natal }\end{array}$} \\
\hline Sim & $42(36,5)$ & $73(63,5)$ & 1,0 & \\
\hline Não & $10(47,6)$ & $11(52,4)$ & $0,63(0,25-1,61)$ & 0,338 \\
\hline \multicolumn{5}{|l|}{ Toques vaginais repetitivos } \\
\hline Não & $09(52,9)$ & $08(47,1)$ & 1,0 & \\
\hline Sim & $43(36,1)$ & $76(63,9)$ & $1,99(0,71-5,53)$ & 0,188 \\
\hline \multicolumn{5}{|c|}{ Apgar do bebê no primeiro minuto } \\
\hline 7 a 10 & $49(39,9)$ & $74(60,2)$ & 1,0 & \\
\hline 6 ou menos & $01(20,0)$ & $04(80,0)$ & $2,65(0,29-24,4)$ & 0,390 \\
\hline \multicolumn{5}{|l|}{ Peso do bebê (contínua) } \\
\hline Média do peso em gramas & \multicolumn{2}{|c|}{$3.179(1.620-4.430)$} & $0,99(0,99-1,00)$ & 0,470 \\
\hline
\end{tabular}

Fonte: Elaborada pelas autoras.

Ser nulípara e relatar número excessivo de toques vaginais ficaram associadas ao relato de realização de episiotomia no modelo final de regressão logística multivariada backward (Tabela 3).

Tabela 3. Análise multivariada dos fatores associados ao relato de realização de episiotomia em parturientes atendidas em um hospital referência do Oeste Catarinense, 2016.

\begin{tabular}{lcc}
\hline Variáveis & OR (IC 95\%) & $\boldsymbol{p}$ \\
\hline Gravidez anterior & 1,0 & \\
Sim (multípara) & $6,46(2,63-$ & 0,000 \\
Não (nulípara) & $15,9)$ & \\
& & \\
Toques vaginais repetitivos & 1,0 & \\
Não & $2,85(0,89-$ & 0,077 \\
Sim & $9,08)$ & \\
\hline
\end{tabular}

Fonte: Elaborada pelas autoras.

As nulíparas (OR $=6,5 ;$ IC $95 \%=2,6-15,9)$ $\mathrm{e}$ as mulheres que relataram toques vaginais repetitivos durante o processo de parturição (OR $=2,8 ;$ IC $95 \%=0,9-9,1$ ) tiveram maior probabilidade de serem submetidas à episiotomia. Esta última associação apresentou um valor boderline p 0,07, porém optou-se pela permanência desta no modelo final em virtude da plausibilidade de sua associação.

\section{DISCUSSÃO}

Neste estudo, não ter gravidez anterior (ser nulípara) e relatar toques vaginais repetitivos apresentaram associação com a episiotomia autorrelatada.

A taxa de partos vaginais (39,9\%) constitui-se em percentual considerado baixo para um hospital público quando comparado aos achados da pesquisa Nascer no Brasil, que descreveu uma taxa de $48 \%$ de partos por via vaginal em todo o país, alcançando uma taxa de $54 \%$ quando analisado o setor público ${ }^{(12)}$.
Constatou-se que a episiotomia e a manobra de Kristeller se destacaram em relação às intervenções realizadas durante o período expulsivo e que, nesse estudo, a taxa de episiotomia $(61,8 \%)$ realizada nas mulheres entrevistadas foi superior a valores encontrados em outras pesquisas realizadas no Brasil $^{(1,13-14)}$ com relatos que variaram de $7,2 \%$ a $56 \%$.

A concepção de que tais intervenções podem se configurar em uma maneira de encurtar o parto ${ }^{(2)}$ ainda é defendida por profissionais que atuam no modelo obstétrico centrado no serviço e no profissional. No entanto, o documento Diretrizes Nacionais de Assistência ao Parto Normal recomenda a não realização de episiotomia de rotina durante o parto vaginal espontâneo ${ }^{(3)}$.

Nesse sentido, discute-se que a prática da episiotomia deve ser restrita e que os profissionais sejam encorajados a utilizar seu julgamento clínico para decidir quando o procedimento realmente é necessário. Pesquisas têm demonstrado que a incisão perineal, durante 
o período expulsivo tem sido aplicada de forma mais criteriosa em algumas instituições, especialmente quando há a participação exclusiva ou majoritária de enfermeiras obstétricas na assistência ao parto ${ }^{(4,13-14)}$.

A diminuição da prática da episiotomia já é uma realidade em países como França, Estados Unidos e Inglaterra. Na França, a taxa de episiotomia em primíparas diminuiu de $71 \%$ em 2003, para 44\% em 2010 ${ }^{(15)}$. Nos Estados Unidos, a taxa de episiotomia foi de $17 \%$ em $2012^{(16)}$. $\mathrm{Na}$ Inglaterra, as taxas variaram de acordo com o modelo de atendimento prestado, correspondendo a $8,6 \%$ em partos assistidos apenas por midwife-led care (enfermeira obstetra), em centro de parto normal, e 19,3\% de intervenções em partos acompanhados por médico e enfermeira obstetra, em unidades hospitalares ${ }^{(17)}$.

A prevalência da manobra de Kristeller na população estudada atingiu percentual superior aos valores relatados em outras pesquisas realizadas com brasileiras, descrevendo desde 9,3\% em Belo Horizonte ${ }^{(13)}$ a $37 \%$ de prevalência em mulheres com risco obstétrico habitual ${ }^{1}$. Os relatos das mulheres entrevistadas indicaram a utilização de uma intervenção danosa e que permanece sendo praticada no meio obstétrico, apesar da ausência de recomendação por parte do Ministério da Saúde e da Organização Mundial da Saúde.

Essa conduta desnecessária e arriscada pode ser considerada uma violência obstétrica por violar o direito da mulher e a sua integridade corporal, uma vez que, além da exposição aos riscos, pode causar à parturiente dor e desconforto durante e após a sua realização(13). As Diretrizes Nacionais de Assistência ao Parto Normal recomendam a não realização da manobra de Kristeller ,no segundo período clínico do trabalho de parto ${ }^{(3)}$. Não existem provas do benefício dessa manobra e, além disso, existem indicativos de que tal intervenção constitui um fator de risco para morbidade materna e fetal(18).

As variáveis ser nulípara e relatar toques vaginais repetitivos, durante o trabalho de parto ficaram no modelo final, estando associadas ao relato de episiotomia. Alguns estudos ${ }^{(1,5-7,14)}$ evidenciaram maior frequência de episiotomia em mulheres que passaram pela experiência de parturição pela primeira vez. Tal associação demanda a necessidade de reforços nas orientações fornecidas às gestantes, durante 0 acompanhamento pré-natal. Nesse contexto, estas mulheres podem não reconhecer serem submetidas à violência obstétrica por desinformação ou informações equivocadas, o que pode acarretar em submissão e aceite de forma consciente ou inconsciente de ações danosas à sua saúde ${ }^{(19)}$.

Optou-se pela permanência da variável relativa a toques vaginais repetitivos no modelo final, pois o excesso de toques pode indicar intervenções demasiadas do profissional de saúde que presta assistência a parturientes, configurando-se como uma violência obstétrica. Dessa forma, se intervenções são realizadas desde o início do trabalho de parto, provável que se mantenham ao longo do processo de parturição. Por ter sido encontrado um $p$ valor boderline, acredita-se que uma maior amostra de mulheres poderia evidenciar uma associação mais forte.

São escassos os trabalhos que mostram a realização de toques vaginais como uma variável associada a ocorrência de episiotomia. Um estudo indicou a realização de toques vaginais repetitivos à violência obstétrica, por ser um procedimento doloroso e sem justificativa clínica ${ }^{(20)}$. Outro estudo mostrou que somente $7 \%$ das gestantes referiram-se à necessidade de receber toque vaginal, durante as consultas de pré-natal $^{(21)}$.

Nesse sentido, torna-se importante compreender que em pré-natais de baixo risco gestacional e/ou em mulheres que não apresentam evidência ou iminência de trabalho de parto prematuro, esse procedimento não se justifica. A modificação de colo uterino está obrigatoriamente atrelada à presença de contrações uterinas regulares, exceto em situações em que, sabidamente, a gestante apresente alguma alteração anatômica do colo do útero e/ou alteração do comprimento cervical e/ou outro achado que indique maior atenção e necessidade de avaliação.

Em virtude do hospital onde foi realizado o estudo ser um campo de prática dos cursos de graduação em Medicina e Enfermagem, bem como residência em Ginecologia e Obstetrícia, acredita-se que o número de toques vaginais excessivos possa estar relacionado à presença desses estudantes e seu processo formativo.

A realização de toques vaginais constitui-se procedimento invasivo, mesmo que útil para averiguação da evolução do trabalho de parto, descida da apresentação fetal, entre outras 
atribuições. No entanto, indica-se a possibilidade de utilização de práticas não invasivas para essa avaliação, tal como a observação da linha púrpura na região sacrococcígea e da expressão corporal da mulher que, geralmente se modificam no decorrer do processo de parturição. Em relação à linha púrpura, pesquisa que objetivou determinar se essa linha pode ser utilizada para estimar a progressão do trabalho de parto concluiu que, mesmo não indicada para ser usada de forma rotineira, apresentou correlação com os parâmetros de evolução do trabalho de parto(22).

A litotomia apresentou-se, inicialmente, no modelo bivariado, um $p$ valor de 0,063 , sendo retirada após controle no modelo multivariado com $p$ valor superior a 0,100 . Considerada uma violência obstétrica ainda praticada ${ }^{(23)}$, outras alternativas têm surgido em substituição à litotômica clássica, principalmente posições verticalizadas como a semissentada, a sentada, a de cócoras e imersão na água ${ }^{18}$. Mesmo assim, o uso da posição litotômica é justificada por alguns profissionais em função de facilitar a visualização do canal de parto e possiilitar a tração do bebê $\hat{e}^{(24)}$. Dessa forma, acredita-se que estar em outras posições que não as litotômicas pode ser um fator de proteção no que diz respeito ao acesso do profissional ao períneo da mulher, visto que a litotomia pode favorecer a realização de episiotomia.

Como limitação deste estudo aponta-se o fato da episiotomia ter sido autorrelatada, porém por se tratar de uma incisão cirúrgica de médio porte e a pergunta ter sido feita em, no máximo, três dias após o parto, acredita-se que as entrevistadas não tenham dificuldade para relatar esse evento. No entanto, reconhece-se que em virtude do procedimento nem sempre ser comunicado à mulher ${ }^{(25)}$, algumas participantes podem acabar apresentando desconhecimento sobre o corte intencional realizado no períneo.

Outra limitação se deve ao tamanho da amostra efetiva da presente análise, que foi de 136 puérperas. Esse número foi um pouco menor do que o número amostral calculado (142) em virtude deste estudo ser um braço menor de um projeto que alcançou a amostra esperada para sua execução (343 puérperas). Embora esta amostra tenha sido menor, as análises estatísticas mostraram intervalos de confiança satisfatórios e a principal variável do modelo final apresentou $p$ $<0,01$.

\section{CONCLUSÃO}

Apesar de esforços no sentido de diminuir as excessivas intervenções, no processo de parturição, a realidade da assistência obstétrica brasileira ainda carece de atenção e profundas mudanças. Estas devem perpassar, principalmente, pela reestruturação dos serviços de saúde, pela mudança de paradigma na formação e pela qualificação dos profissionais que já atuam na assistência às gestantes.

É necessário que intervenções não recomendadas e danosas como a episiotomia e a manobra de Kristeller estejam em consonância com as boas práticas obstétricas e que intervenções realizadas de forma excessiva, como os toques vaginais, sejam evitadas. A manutenção da variável toques repetitivos associada à ocorrência de episiotomia possibilita contribuição ao conhecimento dos fatores de risco para a intervenção, na região sul do Brasil.

Tais mudanças exigem, não só uma necessidade de (re)adequação das práticas técnicas, como também a adoção de uma postura que exige do profissional muito mais o observar antes de intervir e o esperar antes de agir, de maneira que a natureza, sendo respeitada, descarte as possibilidades de intervenções.

\section{REFERÊNCIAS}

1. Leal MC, Pereira APE, Domingues RMSM, Theme Filha MM, Dias MAB, Nakamura-Pereira M et al. Intervenções obstétricas durante o trabalho de parto e parto em mulheres brasileiras de risco habitual. Cad. Saúde Pública. 2014; 30 (1): S17S32. DOI: 10.1590/0102-311X00151513. Disponível em: http://www.scielo.br/scielo.php?script=sci arttex t\&pid=S0102-311X2014001300005

2. Riesco MLG. Nascer no Brasil "em tempo": uma questão de hierarquia das intervenções no parto? Cad. Saúde Pública. 2014; 30 (1): S35-S36. DOI: 10.1590/0102-311XC002S114. Disponível em: https://www.scielosp.org/article/csp/2014.v30su ppl1/S35-S36

3. Brasil. Ministério da Saúde, Secretaria de Ciência, Tecnologia e Insumos Estratégicos, Departamento de Gestão e Incorporação de Tecnologias em Saúde. Diretrizes nacionais de assistência ao parto normal: versão resumida [internet]. Brasília, 2017; [acesso em 09 fev 2018]. Disponível em: http://bvsms.saude.gov.br/bvs/publicacoes/diret rizes nacionais assistencia parto normal.pdf 
4. Carvalho CCMD, Souza ASR, Moraes Filho OB. Episiotomia seletiva: avanços baseados em evidências. Femina. 2010; 38 (5): 1-6. Disponível em: $\quad$ http://bases.bireme.br/cgibin/wxislind.exe/iah/online/?IsisScript=iah/iah.xis \&src=google\&base $=$ LILACS\&lang $=p \&$ nextAction $=1$ nk\&exprSearch=546439\&indexSearch=ID

5. Zhang $M$, Wang $M$, Zhao X, Ren J, Xiang J, Luo B et al. Risk factors for episiotomy during vaginal childbirth: A retrospective cohort study in Western China. J. Evid. Based. Integr. Med. 2018; 11(4):233-241. DOI:10.1111/jebm.12316. Disponível em: https://www.ncbi.nlm.nih.gov/pubmed/30160052 6. Braga GC, Clementino STP, Luz PFN, Scavuzzi A, Noronha Neto C, Amorim MMR. Risk factors for episiotomy: a case-control study. Rev. Assoc. Med. Bras. 2014; 60(5):465-472. Disponível em: http://www.scielo.br/scielo.php?script=sci arttex t\&pid=S0104-42302014000500465

7. Ballesteros-Meseguer C, Carrillo-Garcia C, Meseguer-de-Pedro M, Jordana MC, MartinezRoche ME. Episiotomy and its relationship to various clinical variables that influence its performance. Rev. Latinoam. Enferm. 2016; 24:e2793. DOI: $0.1590 / 1518-8345.0334 .2686]$. Disponível em: http://www.scielo.br/scielo.php?script=sci arttex t\&pid=S0104-11692016000100327

8. Goueslard K, Cottenet J, Roussot A, Clesse C, Sagot P, Quantin C. How did episiotomy rates change from 2007 to 2014? Population-based study in France. BMC pregnancy childbirth. 2018; 18(1):208-12. DOI: [10.1186/s12884-018-1747-8]. Disponível

em:

https://www.ncbi.nlm.nih.gov/pmc/articles/PMC 5987447

9. Mellizo-Gaviria AM, López-Veloza LM, Montoya-Mora R, Ortiz-Martínez RA, Gil-Walteros CC. Frequency of episiotomy and complications in the obstetrics service of Hospital Universitario San José, Popayán (Colombia), 2016. Exploration of maternal and perinatal factors associated with its performance. Rev. colomb. obstet. ginecol. 2018; 69(2):88-97. DOI: 10.18597/rcog.3030. Disponível em: http://www.scielo.org.co/scielo.php?script=sci ar ttext\&pid=S0034-74342018000200088

10. Organização Mundial da Saúde, Saúde Reprodutiva e da Família, Unidade de Maternidade Segura, Saúde Materna e Neonatal. Assistência ao parto normal: um guia prático. Brasília (DF): Ministério da Saúde; 1996.
11. Prado DS, Mendes RB, Gurgel RQ, Barreto IDC, Bezerra FD, Cipolotti $R$ et al. Practices and obstetric interventions in women from a state in the Northeast of Brazil. Rev. Assoc. Med. Bras. 2017; 63(12):1039-1048. DOI: 10.1590/18069282.63.12.1039. Disponível em: http://www.scielo.br/scielo.php?script=sci arttex t\&pid=S0104-42302017001201039

12. Leal MC. Nascer no Brasil: inquérito nacional sobre parto e nascimento. Sumário Executivo Temático da Pesquisa [internet]. Rio de Janeiro: Escola Nacional de Saúde Pública Sérgio Arouca da Fiocruz; s/a. [acesso em 7 fev 2018]. Disponível em: http://www.ensp.fiocruz.br/portalensp/informe/site/arquivos/anexos/nascerweb.pdf 13. Sousa AMM, Souza KVD, Rezende EM, Martins EF, Campos D, Lansky S. Práticas na assistência ao parto em maternidades com inserção de enfermeiras obstétricas, em Belo Horizonte, Minas Gerais. Esc. Anna Nery Rev. Enferm. 2016; 20(2): 324-331. DOI: 10.5935/14148145.20160044. Disponível em: http://www.scielo.br/scielo.php?pid=S1414$81452016000200324 \&$ script=sci abstract\&tlng=pt 14. Vogt SE, Diniz SG, Tavares CM, Santos NCP, Schneck CA, Zorzam B et al. Características da assistência ao trabalho de parto e parto em três modelos de atenção no SUS, no Município de Belo Horizonte, Minas Gerais, Brasil. Cad. Saúde Pública. 2011; 27: 1789-1800. DOI: 10.1590/S0102311X2011000900012. Disponível em: http://www.scielo.br/scielo.php?script=sci arttex t\&pid=S0102-311X2011000900012

15. Blondel B, Lelong N, Kermarrec M, Goffinet F. Trends in perinatal health in France from 1995 to 2010. Results from the French National Perinatal Surveys. J. gynecol. obstet. biol. reprod. 2012; 41: e1-e15. DOI: 10.1016/j.jgyn.2012.04.014. Disponível em: https://www.ncbi.nlm.nih.gov/pubmed/22613118 16. Declercq ER, Sakala C, Corry MP, Applebaum $S$, Herrlich A. Listening to mothers III: pregnancy and childbirth. New York: Childbirth Connection; $2013 . \quad$ Disponível em: transform.childbirthconnection.org/reports/listen ingtomothers

17. Euro Peristat. European Perinatal Health Report: health and care of pregnant women and babies in Europe in 2010. 2013. Disponível em: www.europeristat.com

18. Comissão Nacional de Incorporação de Tecnologias no Sistema Único de Saúde (CONITEC). Diretriz nacional de assistência ao parto normal: relatório de recomendação 
[internet]. Brasília: Ministério da Saúde; 2016. [acesso em 07 fev 2018]. Disponível em: http://conitec.gov.br/images/Consultas/2016/Rel atorio Diretriz-PartoNormal CP.pdf

19. Marchioro D. Conhecimento de primíparas sobre a violência obstétrica [Trabalho de Conclusão de Curso]. Chapecó: Universidade Federal da Fronteira Sul; 2014. Disponível em: https://rd.uffs.edu.br/handle/prefix/1262

20. Tesser CD, Knobel R, Andrezzo HFA, Diniz SD. Violência obstétrica e prevenção quaternária: o que é e o que fazer. Rev. bras. med. fam. comunidade. 2015; 10(35):1-12. Disponível em: https://www.rbmfc.org.br/rbmfc/article/view/1013 21. Mendoza-Sassi, Raúl A, Ulmi AA, Mano PS, Dall'Agnol MM, Neumann NA. Avaliando o conhecimento sobre pré-natal e situações de risco à gravidez entre gestantes residentes na periferia da cidade de Rio Grande, Rio Grande do Sul, Brasil. Cad. Saúde Pública. 2007; 23(9):21572166. DOI: 10.1590/S0102-311X2007000900023. Disponível em: http://www.scielo.br/scielo.php?pid=S0102311X2007000900023\&script=sci abstract\&tlng=pt 22. Nunes RD. Utilização da linha púrpura como método diagnóstico da dilatação cervical e altura da apresentação fetal em gestantes em trabalho de parto [Dissertação]. Palhoça: Universidade do Sul de Santa Catarina; 2017. Disponível em: https://www.riuni.unisul.br/handle/12345/2753

23. Andrade PON, Silva JQP, Diniz CMM, Caminha MFC. Fatores associados à violência obstétrica na assistência ao parto vaginal em uma maternidade de alta complexidade em Recife, Pernambuco. Rev. bras. saúde mater. infant. 2016; 16 (1): 2937. DOI: 10.1590/1806-93042016000100004. Disponível em: http://www.scielo.br/scielo.php?pid=S151938292016000100029\&script=sci abstract\&tlng=pt 24. Santos RCS, Riesco MLG. Implementação de práticas assistenciais para prevenção e reparo do trauma perineal no parto. Rev. gaúch. enferm. 2016; 37(esp): e68304. DOI: 10.1590/19831447.2016.esp.68304. Disponível em: http://www.scielo.br/scielo.php?pid=S1983$14472016000500410 \&$ script=sci abstract\&tlng=pt 25. Pompeu KC, Scarton J, Cremonese L, Flores RG, Landerdahl MC, Ressel LB. Prática da episiotomia no parto: desafios para a enfermagem. Rev. enferm. Centro-Oeste Mineiro. 2017; 7: e1142. DOI: http://dx.doi.org/10.19175/recom.v7i0.1142 Disponível https://pdfs.semanticscholar.org/ff82/9ad261c68 9ffc190f6a7931b52e921422ba6.pdf

Nota: Artigo proveniente da pesquisa base intitulada "Características dos partos em hospital referência de Chapecó-SC", desenvolvida por acadêmicos do Curso de Graduação em Medicina da Universidade Federal da Fronteira Sul (UFFS)-Campus Chapecó/SC. Agradecemos aos acadêmicos Adelaine Marília Pinheiro, Charles Júnior Finco, Karine Bedin e Weder Fernando Nazari pelo apoio na coleta de dados.

Recebido em: 20/02/2019

Aprovado em: 01/11/2019

Endereço de correspondência:

Joice Moreira Schmalfuss

Universidade Federal da Fronteira Sul (UFFS) - Campus Chapecó

Rodovia SC 484 - Km 02 - Fronteira Sul

Bloco dos Professores - Sala 312.

CEP: 89815-899 - Chapecó/SC - Brasil

E-mail: joice.schmalfuss@uffs.edu.br 